\title{
en DETAIL
}

\section{Construire dans l'existant}

\section{Reconversion}

\section{Addition \\ Création}

Christian Schittich (Sous la direction de) M $* 1 \times$ (n)

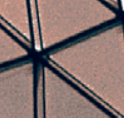



en DETAIL Construire dans l'existant 



\section{en DETAI L}

\section{Construire dans l'existant Reconversion · Addition · Création}

Christian Schittich (Sous la direction de) 
Directeur de la publication: Christian Schittich

Rédaction: Thomas Madlener, Andrea Wiegelmann

Collaboratrices pour la rédaction: Christine Fritzenwallner, Julia Liese

Dessins: Norbert Graeser, Marion Griese, Olli Klein, Nicola Kollmann,

Emese Köszegi, Elli Krammer, Sabine Nowak, Andrea Saiko, Claudia Toepsch

PAO: Peter Gensmantel, Andrea Linke, Roswitha Siegler, Simone Soesters

Traduction: Xavier Bélorgey

Lectorat: André Perret, Ingeborg Seimetz

Cet ouvrage est une coopération des éditions

DETAIL - Revue d'architecture + détails de construction et

Birkhäuser - Verlag für Architektur.

Information bibliographique de la Bibliothèque Allemande

La Bibliothèque Allemande est dépositaire de cette publication dans sa bibliographie nationale; les données bibliographiques détaillées peuvent être consultées sur http://dnb.ddb.de

๑ 2006 Institut für internationale Architektur-Dokumentation GmbH \& Co. KG,

Boîte postale 3306 60, D-80066 Munich, www.detail.de

et Birkhäuser - Verlag für Architektur, Boîte postale 133, CH-4010 Bâle, Suisse,

www.birkhauser.ch

Les droits d'auteur de cet ouvrage sont protégés. Ces droits concernent la protection du texte, de l'illustration et de la traduction. Ils portent aussi sur l'interdiction de réédition, de conférence, de reproduction d'illustrations ou de tableaux, d'émissions radiodiffusées, de microfilmage ou de tout autre dérivé de reproduction ainsi que sur

l'interdiction de divulgation, même partielle, par procédé informatisé. La reproduction de cet ouvrage, dans son intégralité ou par extraits, est liée, même en cas d'exception et par quelque procédé que ce soit aux dispositions légales établies par la Loi fédérale sur les droits d'auteur. Elle est en principe payante. Toute violation de ces droits sera pénalisée selon les dispositions de la Loi fédérale sur les droits d'auteur.

Imprimé sur papier sans acide, obtenu à partir d'une pâte blanchie sans composé chloré (TCF)).

Imprimé en Allemagne

Reproduction:

Karl Dörfel Reproduktions-GmbH, Munich

Impression et reliure:

Kösel GmbH \& Co. KG, Altusried-Krugzell

ISBN 10: 3-7643-7637-6

ISBN 13: 978-3-7643-7637-6

987654321 